\title{
Numerical Simulations of EEG Fields in Three Head Models
}

\author{
L. Peng \\ Mathematics and Science College \\ Shanghai Normal University \\ 100 Guilin Road, Shanghai 200234, China
}

\author{
M.M. Peng \\ College of Informatics \\ South China Agricultural University \\ Guangzhou 510642, China
}

\begin{abstract}
A.H. Xu
State Key Laboratory of Functional Material for Informatics

Shanghai Institute of Microsystem and Information Technology 865 Changning Road 200050, Shanghai, China
\end{abstract}

\begin{abstract}
Electroencephalogrphy (EEG) is a non-invasive method of measuring the electrical activity of the brain. Head model and an efficient method for computing the forward EEG problem are essential to dipole source localization (DSL). In this paper, we use a less expensive nonspherical model to approximate human head. Using this head model, we develop the formulation for computing the forward EEG field by introducing Point Least Square (PLS) method based on Weighted Residual with compactly supported functions. The numerical simulations are implemented in three head models, aiming at investigating the effects of head shape and dipole source parameters on EEG/MEG fields. We also show that PLS method with the trigonometric basis is superior to the constant basis, linear basis, and quadratic basis functions in accuracy and efficiency.
\end{abstract}

Keywords- electroencephalography (EEG); dipole source localization; potential; head model; Point Least Square (PLS)

\section{INTRODUCTION}

Dipole source localization (DSL) has been widely used in analyzing electroencephalograms (EEG's). In order to solve this inverse problem, one must solve the EEG forward problem which is to determine the surface potentials from the dipole current sources in the head volume. For a quantitative interpretation of electroencephalograms (EEG's), a mathematical geometry is needed to approximate the human head. Spherical head models [1] - [3] have been commonly used in EEG problems. However, some key drawbacks are shown using spherical head models in [4],[5]. Nonspherical head models, such as ellipsoid models [5]-[7] and realistic head shape [8]- [10] are also applied. These realistic head models are more adequate to describe the human head than spherical models. However, excessive computing time is required. Therefore such models are not convenient for theoretical analysis. For accurate localization of dipole current source, the information about the effects of the head shape and dipole source parameters on EEG field is necessary. The related work [5],[10] [11] developed some information from different aspects when using spherical or realistically shaped models. In this paper, we use three analytic models(Fig.1(a)-Fig.1(c)), lengthwise ovoid, oblate ovoid model, and sphere to approximate human head aiming at investigating the effects of head shape and dipole source parameters on EEG fields. Point least squares (PLS) based on meshless method for solving EEG forward problem is developed and numerically implemented. Four kinds of basis functions, constant basis, linear basis, quadratic basis and trigonometric basis functions with compact support are applied. We include numerical comparisons of four kinds of basis functions. We organize the paper as follows. Section 2 provides the EEG field equations and our head models. In Section 3, we derive formula of Point Least Square(PLS) for computing the scalp electric potential. The description of numerical experiments is presented in section 4 . In section 5 , simulation results and discussion are presented. In section 6 , conclusion is given.

\section{EEG FIELd EQUATION AND HEAD MODELS}

The typical head model assumes that it is made up of a set of compartments with homogeneous and isotropic conductivity. Let $\Omega$ be the space region occupied by the head and $\mathrm{S}=\partial \Omega$ be the boundary of $\Omega$. Assume that $\Omega$ is divided by surfaces $S_{j}, j=1,2, \cdots M$ into some subregions $\Omega_{j}$ with constant isotropic conductivity $\sigma=\sigma_{j}$ in each $\Omega_{j} \cdot \sigma^{\prime}{ }_{j}$ and $\sigma^{\prime \prime}{ }_{j}$ denote the conductivities the inside and outside of $\Omega_{j}, \quad$ respectively. $\quad \mathbf{x}=\left(x_{1}, x_{2}, x_{3}\right) \quad$ and $\mathbf{y}=\left(y_{1}, y_{2}, y_{3}\right)$ are coordinate vectors in $R^{3}$. EEG forward problem aims at computing the scalp potential that is produced by assumed primary current in the head volume. The relationship between the scalp potential and dipole source can be derived from quasistatic Maxwell's equations [12], that is,

$$
\sigma_{0} V_{0}(x)=\frac{\sigma_{k}^{\prime}+\sigma^{\prime \prime}}{2} V(x)+\sum_{j=1}^{M} \frac{\sigma_{j}^{\prime}-\sigma^{\prime \prime}}{4 \pi} \int_{S_{j}} V(\mathbf{y}) \mathbf{n}_{j}(\mathbf{y}) \cdot \frac{\mathbf{x}-\mathbf{y}}{|\mathbf{x}-y|^{3}} d S
$$


Where $x \in S_{k}, k=1,2, \cdots M, \mathbf{n}_{j}(\mathbf{y})$ is outward directed unit vector normal to the jth surface $S_{j}$ and $V_{0}(\mathbf{x})$ is the potential in a infinite homogeneous medium with conductivity $\sigma_{0}$, that is,

$$
\mathrm{V}_{0}(\mathrm{x})=\frac{1}{4 \pi \sigma_{0}} \int_{\Omega} \mathrm{J}^{\mathrm{p}}(\mathrm{y}) \cdot \frac{\mathbf{x}-\mathbf{y}_{\mathrm{q}}}{\left|\mathbf{x}-\mathbf{y}_{\mathrm{q}}\right|^{3}} \mathrm{dv}
$$

The primary current is generally modeled as a current dipole with moment q located at $\mathbf{y}_{q}$, then we have

$$
\mathrm{V}_{0}(\mathrm{x})=\frac{1}{4 \pi \sigma_{0}} \mathbf{q} \cdot \frac{\mathbf{x}-\mathbf{y}_{\mathrm{q}}}{\left|\mathbf{x}-\mathbf{y}_{\mathrm{q}}\right|^{3}}
$$

The head model we will use is ovoid geometry with surface equation :

$$
S: x^{2}+y^{2}+z^{2}=\left(a^{\frac{1}{n_{0}}} z\right)^{\frac{2 n_{0}}{n_{0}+1}}
$$

Where $a$ is the length of longitudinal axis and $n_{0}$ is called the index.

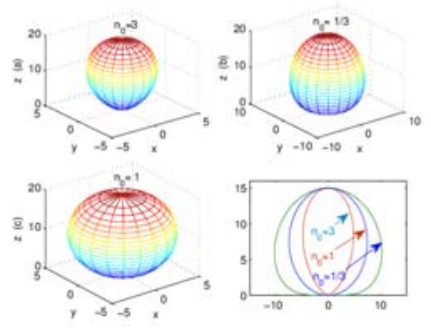

FIGURE I. THREE HEAD MODELS: (A) LENGTHWISE OVOID; (B) OBLATE OVOID; (C) SPHERE; (D)THE OVAL CROSS-SECTION WITH DIFFERENT INDEX.

\section{POINT LEAST SQUARES WITH COMPACTLY SUPPORTED FUNCTIONS}

The techniques widely used to solve the EEG forward problem are the finite element method(FEM)and boundary element method(BEM). FEM for the anisotropic conducting compartments have an increased computational demand [13],[14]. In contrast, BEM for isotropic conducting compartments can reduce much work. But the draw back of BEM is still the computational cost and the singularity in the vicinity of a surface separating two compartments. Most work of BEM is devoted to improving the accuracy [15][17].Currently, the efficiency of EEG forward solutions for non-spherical head models is limited[18]. We use Point of Least Squares (PLS) with compactly supported trial functions. According to the formula (1) we define the operator:

$$
L V=\frac{\sigma_{k}^{\prime}+\sigma^{\prime \prime}{ }_{k}}{2} V(x)+\sum_{j=1}^{M} \frac{\sigma^{\prime}{ }_{j}-\sigma^{\prime \prime}{ }_{j}}{4 \pi} \int_{S_{j}} V(\mathbf{y}) \mathbf{n}_{j}(\mathbf{y}) \cdot \frac{\mathbf{x}-\mathbf{y}}{|\mathbf{x}-y|^{3}} d S
$$

Then the formula (1) is equivalent to $L V=\sigma_{0} V_{0}(\mathbf{x})$. Set the residual error $R=L V-V_{0}(\mathbf{x})$. Let the trial function of the potential be

$$
\hat{V}=\sum_{i=1}^{n} c_{i} \Phi_{i}(\mathbf{x})
$$

Where $\Phi_{i}(\mathbf{x})$ are basis functions, and $C_{i}$ is unknown coefficients. Then the general form of the method of weighted residual can be described as follows:

$$
\int_{S} R w(\mathbf{y}) d \mathbf{y}
$$

Where $w(\mathbf{y})$ is weighting functions. Generally, the weighting function is taken as

$$
w(\mathbf{x})=\sum_{k=1}^{n} \alpha_{k} \psi_{k}(\mathbf{x})
$$

Where $\alpha_{k}$ are coefficients and $\psi_{k}$ are basis functions. Thus inserting each basis function into the formula (7), we have unified form for the method of weighted residuals as follows

$$
\int_{S} R \psi_{j}(y) d y=0, j=1,2, \cdots n
$$

If we choose the weighting functions as $\psi_{j}=\frac{\partial R}{\partial c_{j}}=L \Phi_{j}\left(\mathbf{x}_{i}\right)$, then the general least squares takes the form $\int_{S} R \frac{\partial R}{\partial c_{j}}=0, j=1,2, \cdots n$. Point Least Squares is that we replace the integral by $\operatorname{sum} \sum_{i=1}^{m} R \frac{\partial R\left(\mathbf{x}_{j}\right)}{\partial c_{j}}=0, j=1,2, \cdots, n$, where $\mathbf{x}_{i}$ are node points, and $\mathrm{m}$ is the number of basis functions. Equivalently, we write the matrix form as

$$
\mathbf{T T}^{T} \mathbf{c}=\mathbf{b} .
$$

Where $\mathbf{T}$ is $n \times m$ matrix, $c$ and $\mathbf{b}$ are $n \times 1$ vectors, they are as follows :

$$
T=\left[\begin{array}{ccc}
L \Phi_{1}\left(\mathbf{x}_{1}\right) & \cdots & L \Phi_{1}\left(\mathbf{x}_{m}\right) \\
L \Phi_{2}\left(\mathbf{x}_{1}\right) & \cdots & L \Phi_{2}\left(\mathbf{x}_{m}\right) \\
\vdots & \vdots & \vdots \\
L \Phi_{n}\left(\mathbf{x}_{1}\right) & \cdots & L \Phi_{n}\left(\mathbf{x}_{m}\right)
\end{array}\right], \quad c=\left[\begin{array}{c}
c_{1} \\
C_{2} \\
\vdots \\
C_{n}
\end{array}\right], \quad \mathbf{b}=\left[\begin{array}{ccc}
L \Phi_{1}\left(\mathbf{x}_{1}\right) & \cdots & L \Phi_{1}\left(\mathbf{x}_{m}\right) \\
L \Phi_{2}\left(\mathbf{x}_{1}\right) & \cdots & L \Phi_{2}\left(\mathbf{x}_{m}\right) \\
\vdots & \vdots & \vdots \\
L \Phi_{n}\left(\mathbf{x}_{1}\right) & \cdots & L \Phi_{n}\left(\mathbf{x}_{m}\right)
\end{array}\right] \cdot\left[\begin{array}{l}
V_{0}\left(\mathbf{x}_{1}\right) \\
V_{0}(\mathbf{x} 2) \\
\vdots \\
V_{0}\left(\mathbf{x}_{m}\right)
\end{array}\right]
$$

Where

$$
L \Phi_{j}\left(\mathbf{x}_{i}\right)=\frac{\sigma}{2} \Phi_{j}\left(\mathbf{x}_{i}\right)+\frac{\sigma}{4 \pi} \int_{s_{j}} \Phi_{j}(\mathbf{y}) F\left(\mathbf{x}_{i}, \mathbf{y}\right) d S, \quad F\left(\mathbf{x}_{i}, \mathbf{y}\right)=\frac{\mathbf{n}(\mathbf{y}) \cdot\left(\mathbf{x}_{i}-\mathbf{y}\right)}{\left|\mathbf{x}_{i}-\mathbf{y}\right|^{3}}
$$

It is seen that $A=T T^{T}$ is a symmetric, positive definite and dense matrix. Noting that the surface equation of the ovoid in spherical coordinate system is $S: \rho=a \cos ^{n_{0}} \theta, \quad 0 \leq \theta \leq \frac{\pi}{2}$. And the parametric form is the following

$$
\begin{aligned}
& x=a \cos ^{n_{0}} \theta \sin \theta \cos \phi \\
& y=a \cos ^{n_{0}} \theta \sin \theta \sin \phi \\
& z=a \cos ^{n_{0}+1} \theta
\end{aligned}
$$

We transform the domain of boundary surface integration in Eq.(11) into the rectangular region in spherical coordinate parameters $\theta$ and $\phi$ that is, $D^{\theta, \phi}: 0<\theta<\pi / 2, \quad 0<\phi<2 \pi$. According to the node point 
we divide the surface $S$ into subsurface $S_{j}, j=1,2, \cdots m$. The corresponding domain is sub-rectangular $D_{j}^{\theta, \phi}$. Here we choose the constant basis, linear basis, quadratic basis and trigonometric basis functions with compact support as follows, respectively.

$$
\begin{gathered}
\Phi_{j}(\mathbf{x})=\left\{\begin{array}{ll}
1, & \mathbf{x} \in s_{j} \\
0, & \mathbf{x} \notin s_{j}
\end{array} \quad \Phi_{j}(\mathbf{x})= \begin{cases}1+\theta+\phi, & \mathbf{x} \in s_{j} \\
0, & \mathbf{x} \notin s_{j}\end{cases} \right. \\
\Phi_{j}(\mathbf{x})= \begin{cases}1+\theta^{2}+\theta \phi+\phi^{2}, & \mathbf{x} \in s_{j} \\
0, & \mathbf{x} \notin s_{j}\end{cases} \\
\Phi_{j}(\mathbf{x})= \begin{cases}\cos \phi(\cos \theta), & \mathbf{x} \in s_{j} \\
0, & \mathbf{x} \notin s_{j}\end{cases}
\end{gathered}
$$

According to the parametric form of surface Eq.(12), the collocation points are written as

$$
\mathbf{x}_{i}=\left(x\left(\theta_{i}, \phi_{i}\right), y\left(\theta_{i}, \phi_{i}\right), z\left(\theta_{i}, \phi_{i}\right)\right), i=1,2, \cdots n
$$

Noting that the elements of matrix in Eq.(11) need to handle boundary integrals, which show a strong singularity of the order $r^{-2}$ because of the highly varying kernel $F(\mathbf{x}, \mathbf{y})$ in Eq.(11), we apply boundary regularization method which means that the singular points are replaced by the corresponding points on imaginary boundary surface. Therefore, the formula (11) can be rewritten as

$$
\begin{gathered}
L \Phi_{j}\left(\mathbf{x}_{i}\right)=\frac{\sigma}{2} \Phi_{j}\left(\mathbf{x}_{i}\right)+\frac{\sigma}{4 \pi} \int_{D_{j}{ }_{j}, \phi} \Phi_{j}(\mathbf{y}) \frac{\mathbf{n}(\mathbf{y}) \cdot\left(\mathbf{x}_{i}-\mathbf{y}\right)}{\left|\mathbf{x}_{i}-\mathbf{y}\right|^{3}} \sqrt{E G-F^{2}} d \theta d \phi \\
\text { Where } \sqrt{E G-F^{2}}=a^{2} \cos ^{2 n_{0}-1} \theta \sin \theta \sqrt{n_{0}^{2} \sin ^{2} \theta+\cos ^{2} \theta} .
\end{gathered}
$$

\section{DESCRIPTION OF THE NUMERICAL EXPERIMENTS}

To demonstrate the performance of potentials in different head models versus the varying dipole source parameters, we perform simulations in three head models with indexes $n_{0}=1 / 3, n_{0}=3$ and $n_{0}=1$. The parameters are taken as the following:

$$
\sigma_{0}=1, \Omega^{-1} m^{-1}, \sigma=0.33 \Omega^{-1} m^{-1}, a=19 \mathrm{~cm} d_{0}=0.5 \mathrm{~cm} .
$$

We compute potentials in the following dipole source parameters:

$$
\mathbf{q}=(1,0,0), \mathbf{q}=(0,1,0), \mathbf{q}=(0,0,1), \mathbf{q}=(\sqrt{2} / 2, \sqrt{2} / 2,0) .
$$

The dipole $\mathbf{q}$ was located on $\mathrm{z}$ axis moving from $3 \mathrm{~cm}$ to $15 \mathrm{~cm}$. The forward calculation for EEG is divided into two steps. In the first step, we formulate the matrix T in Eq.(11), which is independent of the parameters of dipole source. We need to compute boundary regularized double integrals in Eq.(16). We implement quadl in dbquadl based on Matlab which is a high order method using an adaptive Gauss/Lobatto quadrature rule. We solve linear systems by LU decomposition. In second step, the potential in infinite homogeneous space which is related to the parameter of dipole source is computed. In order to test the accuracy of our method, we use the relative error as follows

$$
R_{s}=\frac{\left\|\hat{M}-M_{t r}\right\|_{2}}{\left\|M_{t r}\right\|_{2}},
$$

Where $M_{t r}$ is the value based on the approximation of higher order and $\hat{M}$ is the value based on the approximation of lower order.

\section{RESUlTS AND DisCUSSION}

Under the same dipole direction the maps of the potentials versus the sensor position parameter for different dipole position on z-axis are shown in Fig.2-Fig.4. It is shown that their patterns are similar but there is difference in magnitude. The relative differences of potentials versus dipole position for four dipole orientations are seen in Fig.5, which shows they have the same trend. In Fig.6, the peak potentials versus the dipole position in three head models are compared. We can find that the trends of peak potentials as dipole moves toward the surfaces are similar in different head models. The relative differences of potentials computed by four basis functions are presented in Fig.7. It is seen that the relative difference of potential based on trigonometric basis is much better than that based on other three kinds of basis functions. The comparison of the computing time based on four kinds of basis functions can be seen in Table1. It is seen that trigonometric basis has the advantage over other three kinds of basis functions in computing time.

TABLE I. RUNNING TIME (S) FOR FOUR KINDS OF BASIS FUNCTIONS.

\begin{tabular}{|l|l|l|l|l|}
\hline method & const. & linear & quadratic & trig. \\
\hline$n 0=1 / 3$ & 149 & 159 & 161 & 90 \\
\hline$n 0=1$ & 148 & 158 & 160 & 89 \\
\hline$n 0=3$ & 147 & 158 & 159 & 88 \\
\hline
\end{tabular}

\section{CONCLUSIONS}

The analytic head model we introduced is less expensive and convenient to make theoretical analysis. Point of Least Squares (PLS) based on meshless method are applied. Simulations are implemented in four kinds of basis functions for three head models. We obtain the detail information about the effect of head shapes and dipole source parameters on EEG fields. This provides necessary information for accurate localization of dipole current source. In comparison of PLS based on four kinds of basis functions, we find that the trigonometric basis is superior to the other three basis functions in accuracy and in efficiency. It is a significant reference for further computation of EEG forward solutions with anisotropic conductivity head model.

\section{ACKNOWLEDGMENTS}

The work is supported by E-Institutes and Innovation Program of Shanghai Municipal Education Commission (No.E03004 and No.09YZ152). 


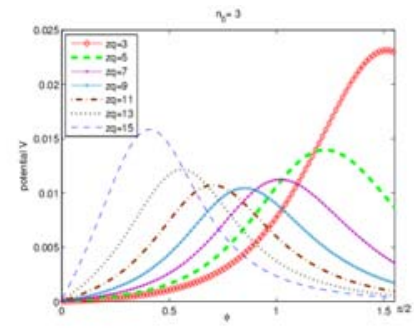

FIGURE II. LENGTHWISE OVOID MODEL. POTENTIALS VERSUS THE ANGLE $\Phi$, THE SENSOR POSITION PARAMETER.

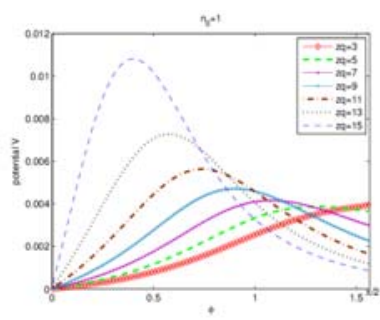

FIGURE III. SPHERE MODEL POTENTIALS VERSUS THE ANGLE $\Phi$, THE SENSOR POSITION PARAMETER.

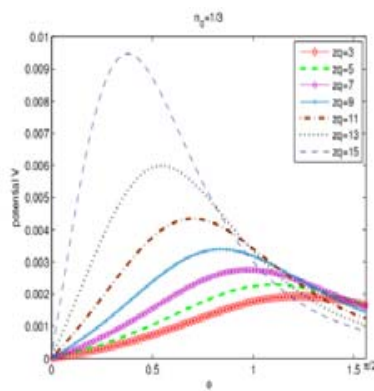

FiGURE IV. OBlate OVOId Model Potentials Versus The ANGLE $\Phi$, THE SENSOR POSITION PARAMETER.

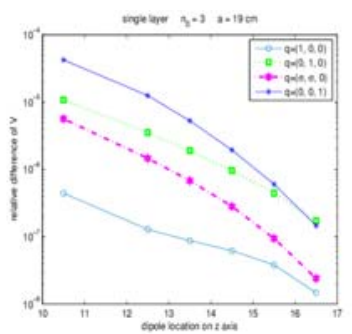

FIGURE V. COMPARISON OF ACCURACY OF POTENTIAL FOR FOUR DIPOLE ORIENTATIONS

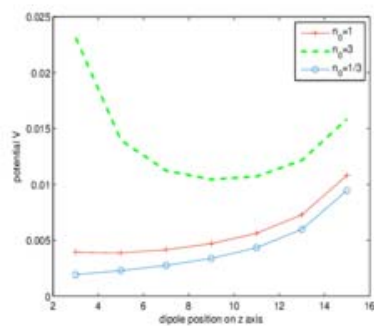

FIGURE VI. COMPARISON OF PEAK VALUe POTENTIALs VERSUS Dipole Position ON Z AXIS IN THREE HEAD MODEL.

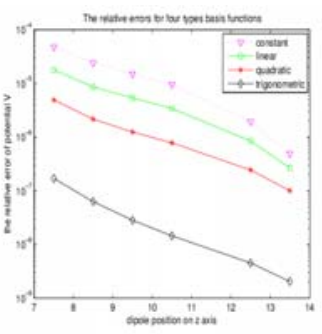

FIGURE VII.

THE ACCURACY OF POTENTIAL BASED ON FOUR BASIS FUNCTIONS

\section{RENFERENCE}

[1] Berg, P. \& Scherg, M., A fast method for forward computation of multiple-shell spherical head models. Electoenceph Clin Neurophysiol, 90,pp. 58-64, 1994.

[2] Zhang, Z, A fast method to compute surface potentials generated by dipoles within anisotropic spheres. Phys. Med. Biol., 40, pp.335349,1995 .

[3] Lalancette, M., Quraan M. \& Cheyne, D., Evaluation of multiple sphere head models for MEG source localization. Phys. Med. Biol.56, pp. 5621-5635,2011

[4] Ermer, J. J., Mosher, J. C., Baillet, S. \& Leahy, R. M., Rapidly recomputable EEGforward models for realistic head shapes. Phys. Med.Biol., 46, pp. 1265-1281, 2001.

[5] Cuffinm, B. N., Effects of head shapes on EEG's and MEG's. IEEE Trans. Biomed. Eng., 37, pp. 44-52, 1990.

[6] Fokas, A. S., Electro-magneto-encephalography for a three-shell model:distributed current in arbitrary, spherical and ellipsoidal geometries. J. R. Soc. Interface, 6, pp. 479-488, 2009.

[7] Gutierrez, D \& Nehorai, A., Array Response Kernels for EEG and MEG in Multilayer Ellipsoidal Geometry. IEEE Trans. Biomed. Eng., 55, pp. 1103-1111, 2008.

[8] Hämäläinen, M.S. \& Sarvas, J. Realistic conductivity geometry model of the human head for interpretation of neuromagnetic data. IEEE Trans. Biomed. Eng., 36, pp.165-171, 1989.

[9] Huiskamp,G., Vroeijenstijn,M., Dijk, G. V., Wieneke,R.\& Huffelen A.V., The need for correct realistic geometry in the inverse EEG problem. IEEE. Trans. Biomed. Eng., 46, pp. 1281-1287, 1999.

[10] Ellenrieder, N. V., Muravchik, C.H., Wagner, M. \& Nehorai, A. Effect of Head Shape Variations Among Individuals on the EEG/MEG Forward and Inverse Problems. IEEE Trans. Biomed. Eng., 56, pp. 587-597, 2009.

[11] Baillet,S., Rivera, J.J., Marin, G., et al, Evaluation of inverse methods and head models for EEG source localization using a human skull phantom. Phys. Med. Biol., 46 pp. 77-96, 2001.

[12] Sarvas, J. Basic Mathematical and Electromagnetic Concepts of the Biomagnetic Inverse Problem. Phys. Med. Biol., 32, pp. 11-22, 1987.

[13] Wolters, C.H., Kuhn, M., Anwander A. \& Reitzinger, S., A parallel algebraic multigrid solver for finite element method based source locationlization in the human brain. Comput Visual Sci, 5 pp.165-177, 2002.

[14] Haueisen, J., Tuch, D., Ramon, C., Schimpf, P., Wedeen, V., George J. \& Belliveau, J., The influence of brain tissue anistropy on human EEG and MEG. Neuroimaging, 15 pp. 159-166, 2002.

[15] Mosher, J.C., Leahy, R. M.,\& Lewis, P.S., EEG and MEG Forward Solutions for Inverse Methods. IEEE. Trans. Biomed. Eng., 46, No.3 pp. 245-259, 1999.

[16] Frijns, J.H.M., de Snoo, S.L. \& Schoonhoven, R., Improving the accuracy of the boundary element method by the use of second-order interpolation functions. IEEE Trans. Biomed. Eng., 47, No. 10, pp. 1336-1346, 2000.

[17] Kybic,J., Clerc, M., Faugeras, O., Keriven,R. \& Papadopoulo, Y., Generalized head models tor MEG/EEG: boundary element method beyond nested volumes. Phys. Med. Biol. 51 pp. 1333-1346, 2006.

[18] Ermer,J.J., Mosher, J.C., Baillet, S. \& Leahy, R.M., Rapidly recomputable EEG forward models for realistic head shapes. Phys. Med. Biol., 46 pp. 1265-1281, 2001. 